\title{
Technical aspects of telepathology with emphasis on future development
}

\author{
P. Schwarzmann, B. Binder and R. Klose \\ Institute of Physical Electronics, University of \\ Stuttgart, Stuttgart, Germany
}

Pathology undergoes presently changes due to new developments in diagnostic opportunities and cost saving efforts in health care. Out of the wide field of telepathology the paper selects three prototype applications: telepathology in teleeducation, expert advice for preselected details of a slide and finally telepathology for remote diagnosis. The most challenging field for remote diagnosis is the application in the frozen section scenario. The paper starts with the mental experiment to map conventional procedures to counterparts in telepathology.

Technical opportunities and economical restrictions of telepathology equipment are discussed with respect to the components: electronic camera, display devices, haptic sensors and displays, available telecommunication channels and telepathology software.

As an example and for illustration of the state of the art for an advanced telemicroscopy system able to perform remote frozen section diagnosis, the HISTKOM equipment is presented in more details.

The section concerning future developments regards the aspects of the acceptance by tentative users, legal aspects, costs and affordability of equipment, the market for equipment components and the adequate telecommunication services. Further is regarded the mutual influence of properties of existing systems and application experiences gained with them on the next generation of equipment and application software. Conclusions and references close the paper.

Keywords: Telepathology, telepathology networks, artificial hand, teleeducation, virtual slide, telemedicine market

\section{Introduction}

The field of pathology presently undergoes worldwide changes as a result of new scientific experiences and tools [3,5], as well as from cost saving efforts:

- Pathology splits more and more into special fields, which make it difficult for the pathologist to overlook still the complete field. This requires an increased number of expert consultations to provide the patient with a the state of the art diagnosis. It requires also adequate opportunities for continuing education to provide pathologists with the results of new developments.

- Due to cost saving efforts, more and more small and medium hospitals lose their own departments of pathology and have to rely on services from outside the hospital.

Both trends make telepathology an attractive tool for requesters and providers of services in pathology to meet the indicated challenges. Accordingly the original motivation for the introduction of telepathology procedures was just to replace the deficiencies mentioned above by the new tool. The basic attraction were two features: (i) to avoid the physical transportation of slides and tissue and (ii) to allow a local separation of the investigated sample and the investigator. The challenge for the developers of telepathology equipment was to consider the new situation and to fit their instruments into the existing workflow in pathology. Later considerations were made, not only to reestablish the traditional workflow, but to exploit additionally new opportunities offered by telepathology.

The present paper will be restricted to the technical aspects of telepathology equipment. It starts in paragraph two with a presentation of different modes of telepathology and their requirements to equipment. In part three, technical solutions together with economical restrictions are discussed and part four demonstrates an example of today's state of the art equipment. Part five finally discusses new opportunities and possible trends. A short section with conclusions and references ends the paper.

To avoid misunderstanding of the term "slide", throughout the paper "slide" will be used only for the glass slides with the prepared tissue for inspection under the microscope, "slides" of photographic films will be called "diapositives". 


\section{What is telepathology?}

The item "telepathology" includes a variety of very different procedures and functionalities in pathology. In principle, it includes everything, provided a telecommunication step is part of a service. A collection and an overview with definitions may be found in a report $[4,14]$ of the EUROPATH project of the EU promoting telepathology and in [8]. For simplicity reasons, we will select three representative telepathology applications and the corresponding equipment covering almost the whole field. These prototypes of telepathology services may be characterized according to their application:

- Education and continuing education;

- Expert advice and 2nd opinion consultation on the basis of a few preselected images of a case;

- Primary telediagnosis including 2nd opinion in the sense of a complete remote diagnosis.

As the item 2nd opinion is used for different procedures: opinion on the basis of a few selected details and opinion in the sense of a 2 nd diagnosis, this item is avoided and replaced in this paper by the item expert advice and telediagnosis for the two procedures.

It should be mentioned here, however, that there is a lot of overlapping between these idealized applications and the real existing equipment functionalities. Also applications not included in the three prototypes are in operation. As an example shall be mentioned the teleevaluation and telequantification of images with image processing methods and tools.

Requirements to the corresponding equipment of the three prototypes will be formulated first just in the light of their aim to replace an already existing service by a counterpart applying telecommunication technology. In a later section of the paper new opportunities emerging from telepathology and their requirements will be regarded separately. In the next section it will be tried to map the existing conventional services identically to their telepathology counterparts. The description of the three prototype services cited will be structured according to the following scheme: description of the application scenario, motivation to apply a telepathology counterpart, technical parameters to map the existing service identical to his telepathology counterpart.

\subsection{Telepathology in education}

In lectures, continuing education and self-education well selected images from microscopic investigations of tissue and cells are presented for illustrations and interpreted by the teacher or the students. Telepathology meets these scenarios, if the images and the auditorium are locally separated. Not regarded within this scenario is the inspection of slides under the microscope, nor the performance of ring experiments with real slides. These scenarios will be covered by the telediagnosis in Section 2.3.

The motivation for telepathology in teaching is to avoid the handling and above all the mailing of diapositives. Instead, the images are collected with an electronic camera by the teacher and stored on an electronic medium within a data base or in a Web-based server data bank. From this server, images may be retrieved via networks of any type and from any location to be displayed to an audience somewhere. This technology makes diapositive collections, their administration and mailing obsolete.

Coming back to the scenario; the only requests to the telepathology technology applied are: the images shall have the same quality as the presentation with the conventional diapositives and the images shall be present for the audience sufficiently fast.

The steps to realize such a scenario include:

- Collection and perhaps annotation of the images by an expert and their digitization;

- Storage and administration in a data bank;

- Facility to transmit retrieved images via networks;

- Receiving and displaying of the images to an audience or to an individual.

To map the diapositive world to the telepathology world, there is only one necessity: to maintain the same image quality as is provided by diapositives. Fortunately the resolutions and field dimensions of color slides correspond approximately to those of modern fields of view in a microscope. According to the technical report of the Zeiss Company (Zeiss, AxioCam, table of technical data of microscope, objectives) a modern microscope objective (e.g., Zeiss Fluar $10 \times$ NA 0.5 ) requires about $4000 \times 3000$ image pixels in a rectangular format to cover the full resolution and field of view. This resolution corresponds with $12 \mathrm{Mbyte} /$ frame data. In principle this resolution should be maintained for all three basic colors red, green and blue. But due to the characteristics of the human visual system, in which color resolution may be reduced compared to the brightness resolution, 12 Mbyte may be equally distributed to the pixels of the three basic colors (for details see Section 3.3 for cameras). For the image contrast a ratio of $1: 256$ corresponds to that of diapositives. 
This amount of data/image has to be regarded for the whole chain: image acquisition, storage, transmission and final display to an observer. The effect of data compression will be regarded separately.

Deviating from the diapositive concept, also a concept known as "virtual slide" is investigated: the aim is to image the whole slide in full resolution to a digital databank, creating a virtual or electronic slide, which may be handled on a display screen similar to a real slide under a microscope. Because the storage of a complete slide in maximum resolution would overload the storage capacities, strategies are applied to store the complete slide only for low magnifications and to restrict the application of high magnifications to areas of special interest, a procedure, which is well adapted to education.

\subsection{Telepathology in expert advice for preselected slide details}

The classical 2nd opinion procedure, which is better described as 2nd diagnosis method, is the sending around of histological slides to colleagues. For this procedure the adequate counterpart is telemicroscopy as described in Section 2.3. Within the context of telepathology, expert advice for preselected slide details is practiced on a larger scale only since net connected computers became available. The classical counterpart was to make some diapositives and to send them to a colleague. This procedure also often is called "passive" telepathology. It should be mentioned however, that if "enough", and, before all, the "adequate" slide details are presented, a diagnosis may be rendered as well.

In this telepathology mode a pathologist selects relevant images of a slide under his microscope and, in advanced versions, collects them in an electronic case folder. Then he sends this folder via electronic mail or another transmission protocol to a colleague. On both sides is a pathologist and the procedure may be completely offline. For this scenario there is no counterpart of a conventional procedure (with the exception of diapositive transfer), as this mode is a child of the computer age. Therefore this telepathology mode is already based on today's standard equipment for cameras, monitors, networks and computers.

The motivation of this procedure is the possibility to ask colleagues for their opinion concerning selected details in a slide and to get their opinion within one or two days.
The technical requirements to these applications have been restricted from the beginning to TV-frame technology, in low quality solutions with FBAS 1-chip cameras and in high quality solutions with 3-chip CCD cameras; only very recently, resolutions beyond the TV-standard become affordable and are considerable for application.

\subsection{Telepathology for telediagnosis}

Telediagnosis offers pathologist an opportunity to diagnose a slide in the same way and quality as if the slide would be under the microscope on their desk.

The most challenging, but also the most attractive, scenario for small and medium hospitals without an own pathologist is frozen section telediagnosis. In this case, during a surgical intervention, tissue for immediate diagnosis is removed as usual. In the conventional procedure the tissue is prepared and diagnosed in large hospitals by the in-house pathologist, or the material is transported to the next pathologist somewhere else, or a pathologist was asked to come to the hospital for the frozen section diagnosis.

The motivation for hospitals without an in-house pathology department to apply telepathology is to avoid the transportation of tissue and the resulting delay in surgery.

The new scenario relying on telemicroscopy avoids this delay: the removed tissue is immediately prepared for frozen section diagnosis in the surgery department, put under the remotely operable telemicroscope. Then a distant pathologist is called by phone and asked for his assistance. If he agrees and owns a display station for telemicroscopy, he is connected to the microscope station, and he may operate it as it would be on his desk and inspect the field of view on a monitor instead of in an eyepiece. He renders his diagnosis and informs the surgery team of the result. Then the connection is released and the pathologist is free to work for another surgery team.

In the case of frozen section service by telepathology however, the change in workflow, compared to the conventional procedure, has to be considered with respect to the distribution of tasks, responsibilities and liabilities. Figure 1 illustrates the workflow for frozen section diagnosis in the conventional procedure (1a) and with the application of telepathology (1b). The main difference is, that the frozen section preparation is now made by the surgery team and no more by the pathologist.

To map the conventional procedure identically to a telepathology scenario the following requests to equipment and technology would be necessary: 


\section{Conventional Frozen Section Diagnosis}

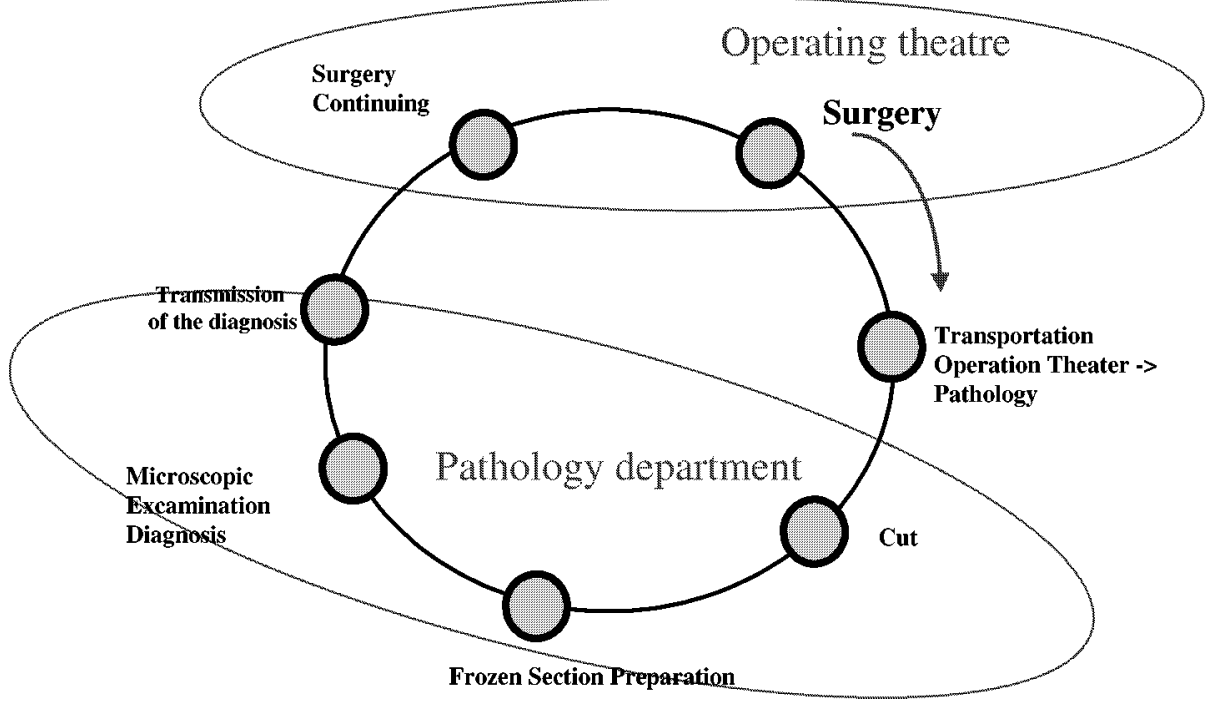

(a)

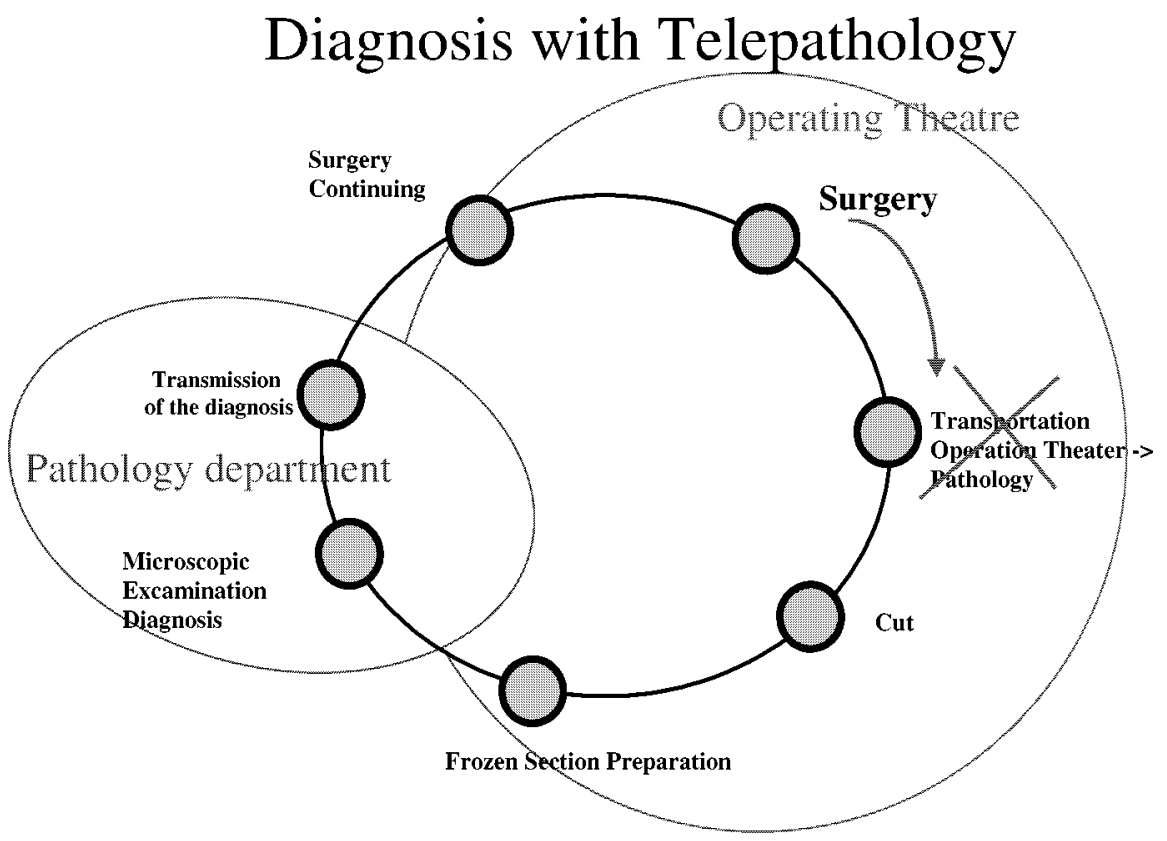

(b)

Fig. 1. The difference in workflow in frozen section diagnosis, aconventional and applying telemicroscopy; (a) is the conventional procedure and (b) applies telepathology. 
- A fully remotely operable microscope (robotic microscope) at the surgery site.

- An online image transmission of the microscope's fields of view to the remote display station of the pathologist to provide the impression of a comfortable interactive workflow with the remote microscope. This is achieved with about 25 frames per second.

- A resolution and field of view of the transmitted images according to those of section 2.1 (4000 $\times$ 3000 pixels/frame).

- A presentation device for the removed tissue as an optical connection during "telepreparation". This is an important component in the frozen section scenario with respect to the main critizism to the introduction of such a service: the preparation of the frozen section slide is made by the surgery team and not by the pathologist. For the pathologist such a macro presentation device is presently the only opportunity to supervise and guide the surgery team in the preparation procedure.

- A haptic device (robotic hand) to feel the tissue for malignant features and for the definition of the position of the frozen section. Additionally, a device to code and transmit the haptic impression and the positions of the robotic finger tips to the remote station of the pathologist.

- A device at the pathologists site to present the haptic information and the finger positions adequately.

- A teleknife to perform the section at the remote tissue.

The complete realization of the above list would provide a "tele frozen section" scenario identical to the conventional procedure.

It is obvious that equipment incorporating all the above features will also be well suited for all other modes of telepathology as, e.g., described in Sections 2.1 and 2.2 - it would become a gold standard.

\section{Technical opportunities and economical restrictions}

Section 3 discusses already existing technical tools to meet the requirements of Section 2. The three prototypic applications will be discussed with respect to three main considerations:

- Technical components and systems available to meet the requirements.
- Economical considerations for the selection of alternatives.

- Technology applied in today's equipment on the market.

Table 1 outlines a list of key components for the three telepathology system types. Also a rough estimation of the availability and affordability is included in the table. The estimation is subdivided into 5 categories:

+ Components in principle available, but make no sense at the moment due to affordability or other reasons; compromises or suboptimal solutions should be accepted.

++ Components available and economically acceptable.

- Solution at the moment not available, but developments are in progress.

- - Solutions not known, and also not visible for the near future.

nr Not relevant.

The remaining part of Section 3 will discuss presently existing solutions for components of Table 1 in more detail with respect to the technical limit, the economical feasibility and the actually implemented solutions in telepathology equipment of the three types. Due to space limitations, only the most challenging

Table 1

Key components of telepathology systems, their availability and affordability

\begin{tabular}{|c|c|c|c|}
\hline Component & Teleeducation & $\begin{array}{l}\text { Expert } \\
\text { advice }\end{array}$ & Telediagnosis \\
\hline Microscopes & ++ & ++ & ++ \\
\hline Microscope stages & $\mathrm{nr}$ & $\mathrm{nr}$ & ++ \\
\hline Cameras & ++ & ++ & $+(-)$ \\
\hline Display screens & $-(+)$ & $-(+)$ & $-(+)$ \\
\hline Host computer equipment & ++ & ++ & ++ \\
\hline \multicolumn{4}{|l|}{ Archiving and memory } \\
\hline capacity & ++ & ++ & ++ \\
\hline \multicolumn{4}{|l|}{ Haptic equipment (artificial } \\
\hline hand and feeling sensor) & $\mathrm{nr}$ & $\mathrm{nr}$ & $-(+)$ \\
\hline Haptic "display" & $\mathrm{nr}$ & $\mathrm{nr}$ & -- \\
\hline Teleknife & $\mathrm{nr}$ & $\mathrm{nr}$ & + \\
\hline \multicolumn{4}{|l|}{ Telecommunication } \\
\hline channels & ++ & ++ & + \\
\hline System software & ++ & ++ & ++ \\
\hline Telepathology software & ++ & ++ & ++ \\
\hline
\end{tabular}


components are discussed, all other components are without problems, available off the shelf and well suited for telepathology applications.

\subsection{Cameras}

The camera is the first member of the image transmission chain: camera - telecommunication link - display device. It determines together with the other members the quality and therefore also the aptitude for telepathology. As already mentioned in Section 2, the field of view and the resolution of modern microscopes and that of diapositives determine the gold standard for a telepathology scenario, which is fully compatible with the conventional procedure in education and diagnosis. Expert advice telepathology makes an exception, as this mode had no equivalent with a conventional procedure and the quality of TV-images was accepted from the beginning. Therefore the requests to expert advice telepathology are satisfied with high quality TV-color cameras of the 3-chip-type. Only recently it was suggested to use cameras with higher resolution and field of view. The aim of these efforts is to reach the xsame resolution and field of view that modern computer screens (about $1600 \times 1200$ pixels/image) provide.

The other modes teleeducation and telediagnosis, however, orient themselves at the microscope and the diapositive resolution and end up at $4000 \times 3000$ pixels/frame. For images with such pixel resolutions, today's camera technology offers several opportunities:

(1) Cameras with sensor chips of such a resolution and field of view. Upper end electronic photo cameras meet these requirements. These cameras can therefore directly replace the diapositive photocamera on the microscope, because their sensor has also about the format of a diapositive and because these sensors are embedded in standard photo camera cases. A critical point for the user is to know, to which degree the images at the output interface of the camera are not only compressed but potentially also reduced in the information content. Such reduction procedures are applied often to save memory capacity! In our application the full information should be available at the camera-computer interface.

Another point to be regarded is the handling of the color information. Only 3-chip cameras or sequential color filtering of 1-chip cameras provide RGB-data of the identical object location. Masks in front of the sensors with color patterns (RGB or Complementary Color Mosaic) provide a reduced spatial color resolution. Figure 2 illustrates the main concepts of color representation for cameras used presently.

The grey value resolution (contrast) corresponds to that of the color diapositives 1:256 (256 grey levels) and is determined by the signal to noise ratio (SNR) of the CCD-pixels. The contrast may be increased by cooling the sensor or by applying averaging to improve the SNR.

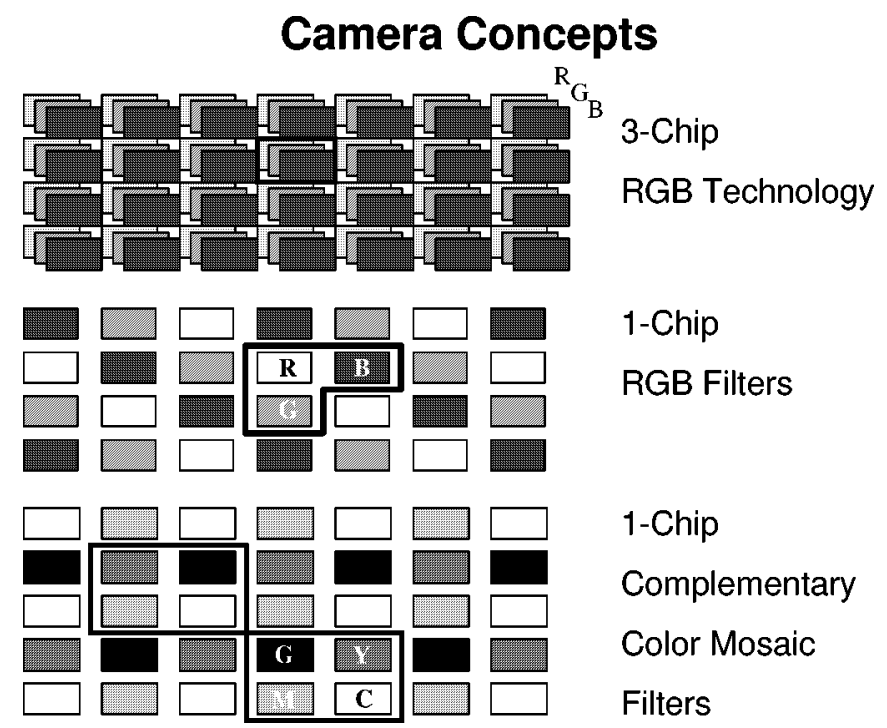

Fig. 2. The main concepts presently used to represent colors in electronic cameras. Top panel: 3-chip-RGB representation (corresponds to the sequential acquisition of three color images), middle panel: RGB color mosaic mask, bottom panel: complementary color mosaic. 
Other technologies with even higher spatial resolutions are scanning line cameras, in which one coordinate direction is scanned with a scanning mirror.

The new upcoming CMOS-sensor technology with its attractive opportunities to integrate image preprocessing steps on the sensor chip to realize, e.g., nonlinear characteristics and to realize contrast figures beyond $1: 100000$ are announced by the Foveon Company in the year 2000, but not yet on the market for capacities larger than $1024 \times 1024$ pixels/frame [9]. These cameras are interfaced to the computer via special digital interfaces, new versions will use the standard USB (Universal Serial Bus).

(2) Sensor-Shift-Technology cameras. This technology applies conventional TV-CCD sensors with reduced fill-factors of the light sensitive area. Between a series of sequentially acquired images the complete sensor chip is moved within subpixel distances and at the end of the image acquisition procedure all subimages are combined (see Fig. 3 for illustration).

Applying normal TV-CCD-sensor chips with $768 \times$ 576 pixels/frame and a reduction of the sensitive pixel aperture to $1 / 5$ of the pixel distance, the combination of 25 subimages results in a combinatorical image of about $4000 \times 3000$ pixels/frame. Representatives of this camera type are the ProGes camera from Jenoptik, Jena, the AxioCam from Carl Zeiss, Oberkochem, and the Nikon DXM 1200, Nikon, Tokyo. The principle and the system theoretical aspects of which have been published by Lenz [7]. Color is introduced by a color mask in front of the sensor chip. The drawback of this technology is a reduced light sensitivity due to the low fill-factor and a relative long image acquisition time due to the necessity to get a series of images for one result image.

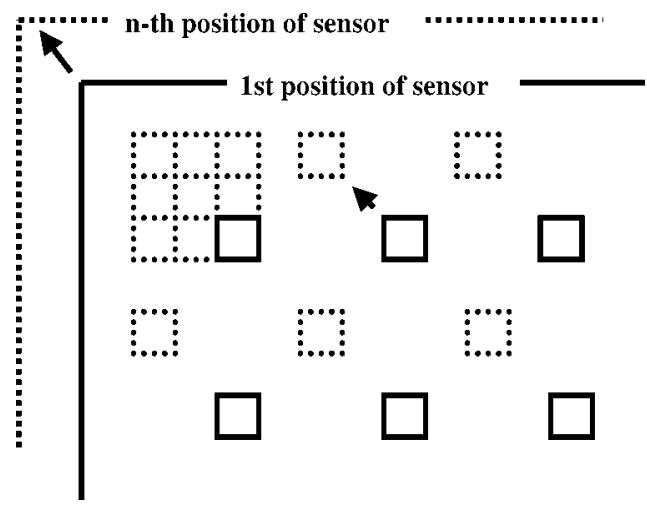

Fig. 3. The shift-chip technology to achieve high resolution with standard TV-technology sensors.
(3) The tiling of images. Histological slides are static objects and can therefore be scanned as subimages (tiles), which later can be combined appropriately to one single large image. This technique can be performed with the standard TV-cameras acquiring TVimages with $768 \times 576$ pixels/frame. After acquiring an image the scanning stage of the microscope is moved by one image distance. To get a perfect fit at the tile borders either the scanning stage has to be moved with pixel accuracy or overlapping image tiles must be collected, which are then fused to the combination image [11]. With this technology in principle any dimension of images can be achieved.

In conclusion: there are several technologies available to collect images with the characteristics of diapositives as far as the image format and resolution is concerned.

For online telediagnosis additional to the image format the frame rate is an important key feature. As already stated, the gold standard for an online transmission would be the video frame rate -25 frames $/ \mathrm{sec}$ - to provide a comfortable impression for moving objects.

All high resolution cameras mentioned above do not meet the online requirement. For the digital photo cameras we find normally a transfer interval of several seconds between two full frame images. The product family for online image acquisition is not the digital photo line but the video camera sector. For online video applications we find here presently video cameras for HDTV applications. High quality 3-chip CCD video cameras for full resolution RGB-signals are on the market with about $1600 \times 1200$ pixels/frame and 25 frames/sec. This technology is below our gold standard for lateral resolution but fits to the data of modern high resolution computer monitors (see also Section 3.2). We will see later (Section 3.6) that even this format will require transmission capacities not affordable today.

The question is, which of the solutions are acceptable and affordable for the different telepathology modes. For teleeducation and expert advice, online capabilities are not relevant and only the resolution must be regarded. The extremely high resolution cameras with $6000 \times 4000$ pixels are very expensive (>20000 €) and therefore only affordable for a few applications but not for making slides in pathology. The shift-chip cameras also cost about $10000 €$ and come near to High Density TV-oriented cameras as, e.g., the JVC type KYF-70 from the Victor Company, Tokyo $(1360 \times 1024$ pixels $)$. The cheapest solution provides the tiling of large images, if there are no other reasons not to use this technique. But remember for a 
tiling without image fusion at the image borders, high precision scanning tables must be installed.

As most of the systems used in education have been designed by the users themselves, who are normally not willing to invest too much labor in design and construction of systems, which would also fit the necessities of a broader market, we find only a few commercial systems on the market today, fulfilling the slide quality request. In most cases the users restrict themselves to the application of the normal TV-camera resolution. There is a tendency today to use digital photo cameras as they become more and more introduced in the consumer market with decreasing costs and rising quality.

For telediagnosis, due to the limited capacity of the transmission channels, exclusively 3-chip CCD TV-format cameras are implemented (about $5000 €$ ). Only for in-house applications with own broadband TV-networks HDTV cameras make sense.

\subsection{Display devices}

The counterpart to the camera equipment is the image display facility at the location of the remote partner. Presently two technologies are commonly used:

- The conventional tube monitors;

- LCD monitors (Liquid Crystal Display).

Other technologies are found only in niche applications:

- Plasma display screens;

- LED-screens;

- Video beamers for a greater audience;

- Laser scanner displays.

For the well known and long introduced tube technology we find today resolutions until 2000 pixels/line and for LCD displays the limit presently is at 1600 pixels/line. Both types allow frame rates beyond 25 frames/sec but do not meet the request for $4000 \times 3000$ pixels/frame; they are however compatible with the HDTV camera format. Both technologies operate with a contrast ratio beyond $1: 100$, which is adequate for 8-bit data.

Presently the image quality of tube displays for natural image scenes is regarded still better concerning the viewing angle and the linearity of the intensity. On the other side LCD displays have a tremendous advantage with respect to geometrical distortions, weight, dimensions and power consumption.
Image dimensions beyond those mentioned above can be realized with laser scanning monitors and, as the counterpart of the tiling technology at the acquisition site, by the presentation of the image simultaneously on several monitors side by side. Unfortunately the borders of tiles stay visible.

Due to the limited potential to reduce further the size of the image pixels on the screens, an adequate distance of the observer to the screen is mandatory, not to see artifacts from the screen structure, a recommendation, which is often violated.

\subsection{Haptic equipment}

This and the next two sections are only relevant for telediagnosis and especially for the frozen section scenario. Until now the haptic information, which is important to locate the section in a tissue correctly, is the only information which is presently not transmitted with telepathology equipment to the remote pathologist. This fact is the main reason for hesitations to introduce telepathology services on a broader basis. Presently, the surgeon is responsible to determine the location for the section. He may be guided by the remote pathologist in this task with a telecursor in the macro-image of the removed tissue in the "presenter", a module, which is offered by most of modern telepathology systems.

We find surgeons in a similar situation, when they switch from conventional surgery to minimal invasive surgery - they also miss the direct haptic contact to the tissue they operate on. It is therefore also a basic concern of surgeons to get a tool for "telefeeling". Another stimulation to develop such instrumentation comes from the industry, when robots have to work with delicate objects in for humans hazardous environments.

Having in mind these other applications, the "telefeeling" aspect becomes much less futuristic, as the pressure to promote this field is strong enough to push such developments.

Today prototypes of artificial hands with the same kinematics and geometry as the human hand already exist [13]. Such devices grab objects in a similar manner as humans do. Figure 4 illustrates such a device. Presentations to grab a raw egg look very promising. The way to market for such devices should not be too long from now.

Quite different is the situation for the sensory part of such a module, where not only forces have to be transmitted but feelings as, e.g., surface characteristics 


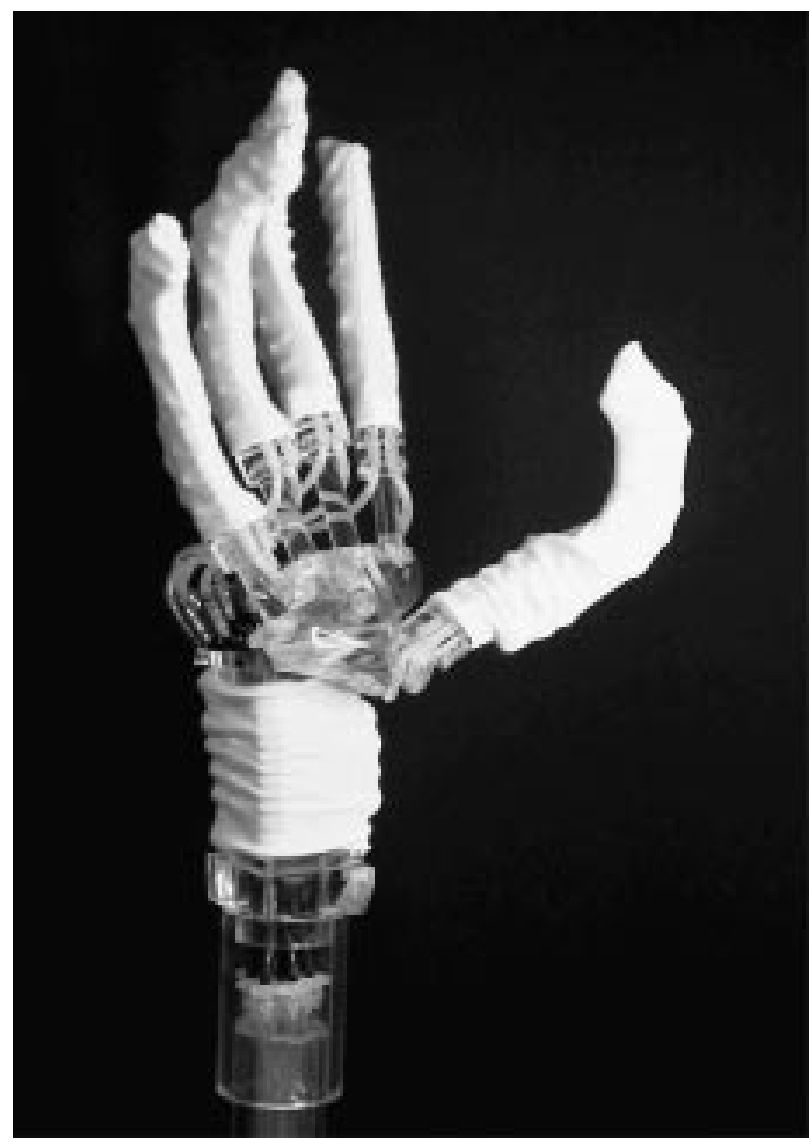

Fig. 4. Example for a prototype of an artificial human hand (by courtesy of S. Schulz, Forschungszentrum Karlsruhe).

like: rough, smooth, dry, soapy, etc. In this case we are by far more away from solutions for technical sensors with similar qualities as human fingers, than in the kinematics model.

The necessity for haptic equipment is discussed controversially in different circles of pathologists. One fraction asks for such equipment to keep the frozen section procedure under the full control of the pathologist, and the other fraction agrees to delegate the task to the surgery team under visual control of the pathologist.

\subsection{Haptic displays}

The counterparts of the haptic actors and sensors are the corresponding tools at the remote site as an interface to the human hand. Like stated above some acceptable solutions for the kinematics capabilities of the hand using a force feedback exist (manipulators, electronic gloves) and are applied in handling machines for hot cells in nuclear fuel plants. They allow an excel- lent handling of bottles, tools, etc. and allow the remote classification of hard and soft objects. But in total they are far from the more complexcapabilities of the human hand. The technology is applied in telesurgery and the related minimal invasive surgery.

Nearly no solutions exist to the authors knowledge to reestablish feelings as indicated in 3.3 by a mechanical presentation device to the human skin of the fingers. What is known is a solution for blind people to feel letters or symbols acquired by a camera and transformed into a topologic object by an array of small differently vibrating pins.

\subsection{Teleknife}

To allow the remote pathologist to make the frozen section cut of the tissue, he needs a tool called teleknife. As already indicated in Sections 3.3 and 3.4 solutions exist and are in operation in robotics and in telesurgery. Under visual control the comfort of these tools is acceptable. 


\subsection{Telecommunication channels}

The characteristics of the telecommunication channel determine to a high degree the operational comfort of a telepathology system. This is obvious for all online services, but also influences expert advice and teleeducation services. To get an impression of the scale of information content and the corresponding intervals for data transmission, we regard 3 mile stones:

- Assuming our ideal image format of 12 Mbytes/ image and an online frame rate of $25 \mathrm{images} / \mathrm{s}$, we end up with a data flow rate of $2.4 \mathrm{Gbit} / \mathrm{s}$ !

- Assuming only a color TV-image format of $768 \times$ 576 pixels and a ISDN telephone line with 64 $\mathrm{kbit} / \mathrm{s}$, the transmission time for one frame consumes about $2.7 \mathrm{~min}$.

- Telecommunication service providers charge customers with respect to several criteria:

* Amount of data transmitted;

* Connection time and connection distance;

* Channel capacity requested;

* Quality of service requested (e.g., online or offline connection).

From a technical point of view $2.4 \mathrm{Gbit} / \mathrm{s}$ are state of the art and are easily transmitted by modern glass fiber connections. The bad news are that $2.4 \mathrm{Gbit} / \mathrm{s}$ correspond to about 37000 telephone channels with the corresponding fees, which become really unaffordable if requested from public service providers. The situation may be much more favorable, if glassfiber lines are available within an institution.

Economically realistic concepts will therefore have to consider limitations in the data transmission capacity. Another limitation will be the restriction to services, which are available everywhere and also used for different purposes than telepathology. In principle we have to restrict to following services:

- Analog telephone (ca. $3.5 \mathrm{kHz}$ bandwidth).

- ISDN lines with $2 \times 64 \mathrm{kbit} / \mathrm{s}$.

- ATM connections (rarely available for end users).

- UMTS mobile phones with $2 \mathrm{Mbit} / \mathrm{s}$ (a new, not yet available but coming network).

- Internet connections (normally limited by the service provider to the telephone channel capacity).

- xDSL services from ISDN-data rate to about $1000 \mathrm{kbit} / \mathrm{s}$ download bandwidth, but the connections are asymmetric: download is normally about 5 times faster than upload.

- In-house connections.
For expert advice telepathology the data rate and the online capacity are of minor interest as store and forward operation is performed. For these services the cheap Internet is the preferred network. The same holds for teleeducation services, if images are retrieved from remote data bases in advance of a presentation. If they are retrieved from a remote database online during a presentation, delay times of more than 5-10 s become unacceptable. As indicated above telephone lines are too slow for this application.

For online telediagnosis in frozen section scenarios $n \times$ ISDN connections ( $n=6-10)$ are the most appropriate selection today, which may be replaced in the near future by the upcoming UMTS device generation. This is a reasonable compromise between comfort and affordability. To reach a sufficient speed in telediagnosis workflow considerable efforts are necessary to manage the data flow appropriately. The most important procedures to reduce the data flow and a rough estimation of the data reduction effect are listed below:

- Reduction of the image format from a diapositive to a color TV-frame format: $50 \times$;

- Data compression without visible loss: $5 \times$;

- Reduction of frame rate: $3 \times$;

- Transmission of only novel image details: $10 \times$;

- Introduction of local intelligence, e.g., for autofocus, autoillumination: $3 \times$;

- Permanent slide overview image for orientation: $3 \times$.

Accumulating the performances of the procedures cited, we get a data reduction of about $70000 \times$. Applying this reduction to the $2.4 \mathrm{Gbit} / \mathrm{s}$ data flow, results in a reduced data flow of under $0.5 \mathrm{Mbit} / \mathrm{s}$. This meets about the capacity of 8 bundled $64 \mathrm{kbit} / \mathrm{s}$ ISDN channels. Data reduction procedures are discussed in the software Section 3.7.

Another aspect of the data transmission channel is data security and data confidentiality. These functions may be part of the network or may be part of the system software. Network providers offer solutions based on intranets requesting identification as well as authentification for the access to the net. Cheap but not very secure means are simple passwords, better solutions rely on chipcards and keys of certified trust centers for encryption.

In conclusion: The state of technology imposes no restrictions to the data flow requested. Limitations result from economic reasons. The limited data rates cannot provide the workflow speed of conventional frozen section services but they provide an acceptable level, 
offering a routine application even in the most challenging telepathology mode - the online frozen section service.

\subsection{Telepathology software}

The telepathology software integrates computer equipment and peripheral devices into a telepathology tool. It determines the quality and the comfort of a telepathology application.

As already indicated in the introduction, teleeducation and expert advice telepathology are present with a wide spectrum of software with different degrees of quality and operating comfort. Commercial software for these applications meets requirements concerning safety of operation and adaptation to the workflow in these fields. Compared to hardware investments in addition to already present equipment, these software modules are expensive and therefore often self written modules are applied between two users.

Quite different is the situation in telediagnosis. In this case the investment for new equipment is high (computer controlled microscope, camera, framegrabber, network modules, etc.) and the software necessary to operate adequately such equipment is complex. Telediagnosis is therefore dominated by certified commercial software systems. All manufacturers of equipment provide their own software, but nevertheless some basic modules more or less common to most systems can be identified:

- System software integrating and supervising all hard- and software modules.

- The user interface, which provides the tools to operate the system and which displays image and other information in an adequate manner to the user.

- The data management module is responsible for adequate data reduction, transmission and presentation.

- The documentation and archiving module meets requirements of legal necessities and exchange of case oriented patient folders.

- Macrofacilities provide means for the pathologist to guide the surgery team during the frozen section procedure.

- Teleconference modules improve telepresence during sessions.

- In future, image evaluation tools and expert system modules will further improve the diagnostic procedure.
Good software concepts allow with acceptable efforts the adaptation to new applications and workflows in telemedicine.

\section{HISTKOM as an example for an advanced telediagnosis system}

A collection of examples documenting the state of the art of today's telepathology technology and applications in general may be found in [6]. Telepathology systems on the market iclude CORABI from Apollo Systems, USA, HISTKOM from the Deutsche Telekom AG, Germany, MIGRA from Olympus, Tokyo, Japan, TPS from Leica Microsystems, Germany, the Zeiss LINK from Tripath Imaging + Carl Zeiss, Germany, or ZEM from Nikon, Japan. his section presents representative also for other developments [21] of telemicroscopy equipment an overview of the HISTKOM [5] telepathology system for telediagnosis. The key design criteria are similar, and can be described for the HISTKOM system as followshave been:

- The system must be able to perform all modes of telepathology, especially online frozen section diagnosis.

- The device must meet legal necessities as documentation of sessions, safety and confidentiality of data.

- The system must rely on networks everywhere available and affordable.

To keep this section short, the functional description of the system is condensed in the agenda of Fig. 7 for the user interface and Fig. 5 presents the equipment in the surgery department, the microscope station. Figure 6 illustrates the equipment at the pathologists site, the display station.

The functionality of the HISTKOM equipment has been evaluated in more than 10 fieldtests. These fieldtests were designed to test the diagnostic quality with retrospective representative embedded and frozen section material, in routine online clinical frozen section operation and in tests for telecytology (PAP-smears).

\section{Future development}

From today's point of view it is difficult, if not impossible at all, to make reliable prognostications for the future development of telepathology and its equipment. With some assumptions nevertheless at least 


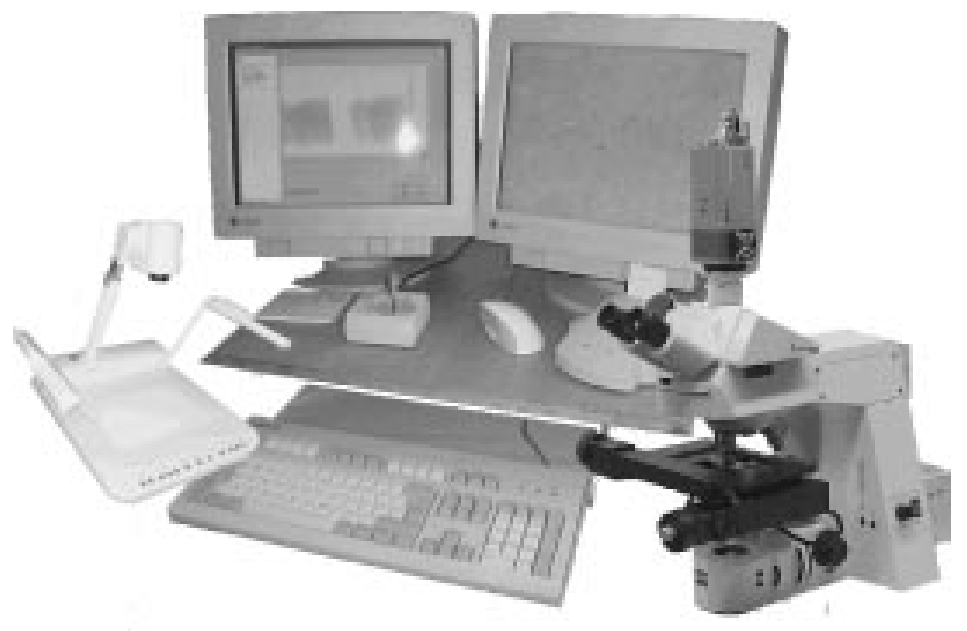

Fig. 5. HISTKOM microscope station with the remotely controllable microscope with scanning table and color TV-camera, the macro device to present the removed tissue before section and to guide the surgery team in frozen section preparation; The host computer; the two monitors display the user interface and the field of view of the microscope; the monitors display identical information as those at the pathology display station and are used also in conference mode.

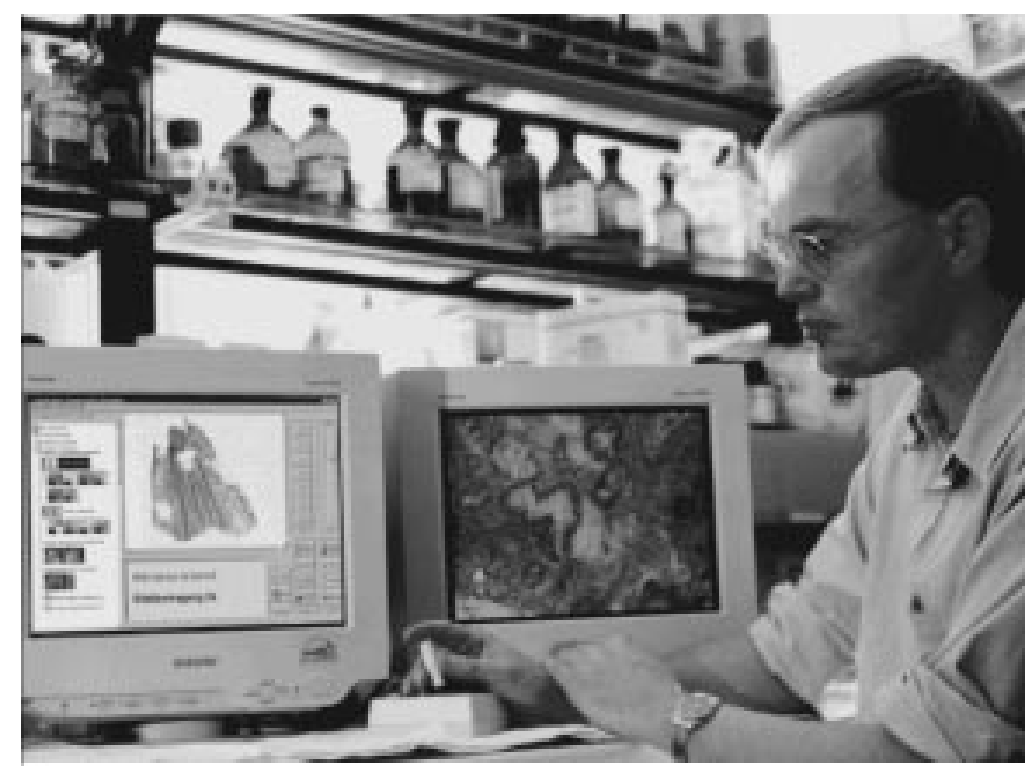

Fig. 6. The remote display station in the pathologists lab consists of 2 monitors displaying the identical information as on the surgery site, the host computer and a joy stick to operate alternatively to the mouse the scanning table.

some general considerations can be made. Important aspects identified already, which will influence the future development, will comprise different fields:

(1) The acceptance by and the requests of the community of tentative users of telepathology services.

(2) The alterations in routine workflow and the legal aspects derived from it.
(3) The tradeoff between requested type and quality of telepathology equipment and the costs accepted for equipment and fees.

(4) The development of the component market for telepathology equipment and of the market of telecommunication services.

(5) The progress of equipment and service quality due to mutual influences resulting out of operational telepathology services. 


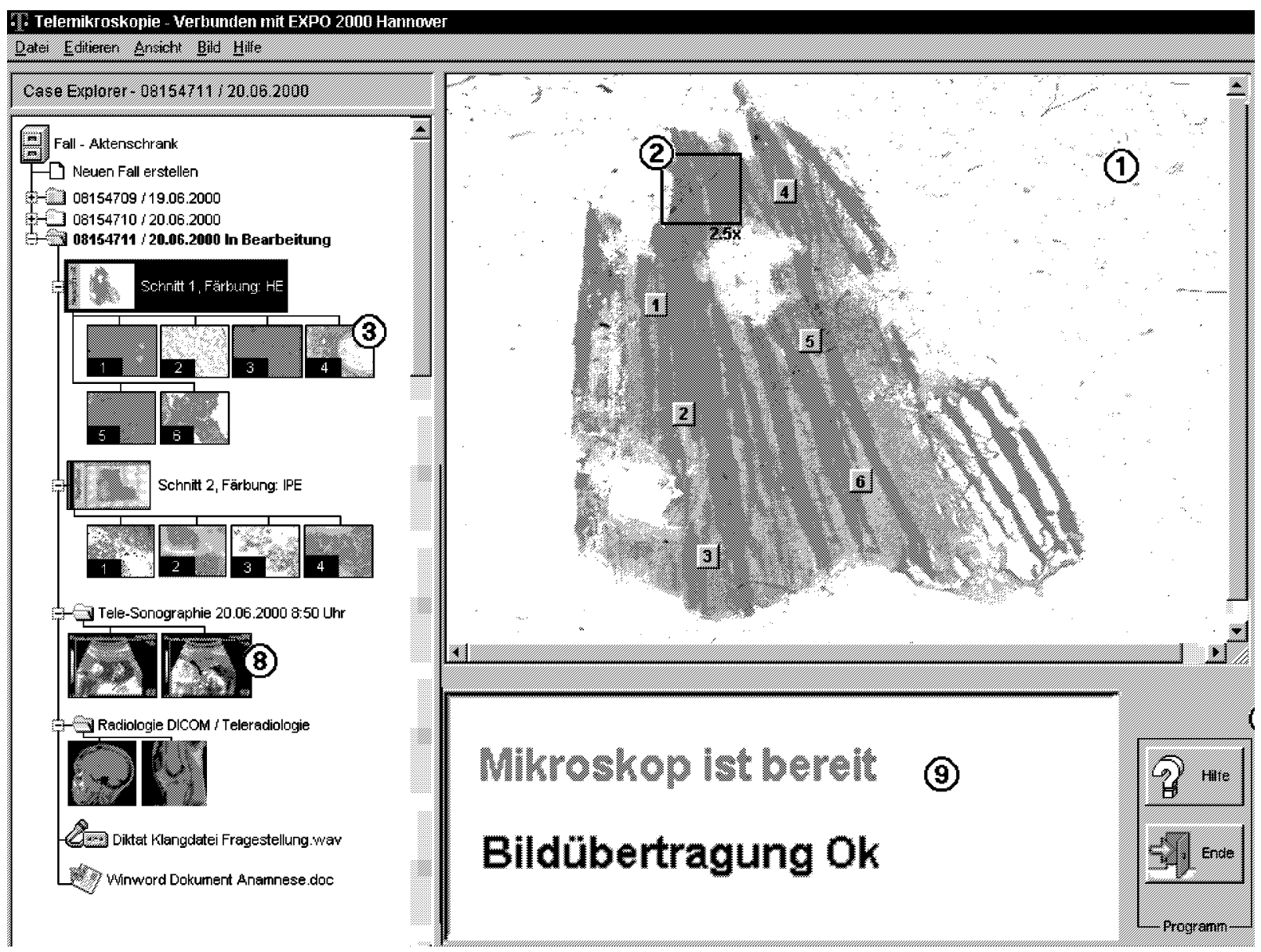

Fig. 7. The user interface in detail with the description of the functionality. Out of the complete functionality a subset is listed below using the labels in the image. 1: Large overview of the total slide in high quality and resolution. 2: Window indicating the position and the scale of the actual microscope field of view displayed on the other screen. 3: Overview of positions of all screen shots of key scenes made and archived in the investigation protocol during the diagnostic session. 4: Buttons to select the objectives respectively the magnification of the microscope. 5: Control to set the microscope illumination. 8: Buttons to call other image material (e.g., US-imagery) of the patient folder or of another camera (e.g., video conference camera) on the screen. 9: Field displays information of presently active functions on the remote microscope and their status (e.g., busy (wait) or ready to accept new instructions). This field also accepts and displays text as patient information, diagnoses and recommendations. 10: Button to request an autofocus procedure on the microscope. 11: Interactive control of the focus position (e.g., for focusing through a nucleus). 12: Button to request a zoom activity at the microscope (change of objective by one step and request of an autofocus activity).

Future development considerations will be discussed in the following according to the list above, but will not repeat considerations already made in Section 3.

\subsection{The acceptance of telepathology by an interested community of users}

Today two motivations with completely different intentions to apply telepathology are found:

- An exchange of experience between pathologists, who know each other well. Expert advice consultation and store and forward technology are the characteristics of this type of telepathology. The procedure normally is not included in a normal routine workflow.

- The other motivation results from a request of non-pathologists for services in pathology. The service asked for is telediagnosis including its most challenging mode of online frozen section services for institutions without an own pathologist. The initiative for this applications often comes from surgery teams and hospital administrations and not from pathologists.

The service is intended for the routine daily workflow of patient treatment and therefore contains 
considerable economical aspects. Such services can only be operated with certified professional equipment, a high quality of service and the acceptance of the corresponding costs.

The motivation for the second type of telepathology (telediagnosis) will promote the development of networks with participants requesting and participants offering services in pathology or probably even all types of services in telemedicine. Figure 8 illustrates this situation.

Such networks, one day when they exist, will also host the expert advice and continuing education services but with professional and standardized hard- and software to maintain interoperability between all partners in the network. In the very beginning, islands will form as is the case today, but if a critical number of participants and telepathology applications exist, these islands will condense to more general telepathology or telemedicine networks. They will provide an electronic market place for telemedicine services and documents (e.g., electronic patient folders of investigations, accounting documents, protocols of remotely performed evaluations of image material, etc.), which allows the full exploitation of the opportunities of telemedicine.

An example to test the acceptance of a routine telepathology service is the EUROQUANT service server of the University of Dresden, which has been developed by financial support of the EUROPATH European Union, project (DG XIII-Project HC 1038). EUROQUANT is a subproject within the EUROPATH. This service provides clients, which send in images of Feulgen stained cells of cytological imprints, with DNA histograms and their interpretations including quality check of the material sent. It is an example of a new upcoming service interpreting remotely images with image processing tools. The service is one of the outcomes of the EUROPATH project of the EU.

The tendency to start professional telemedicine networks becomes already visible by several start-up companies which offer today at least a limited amount

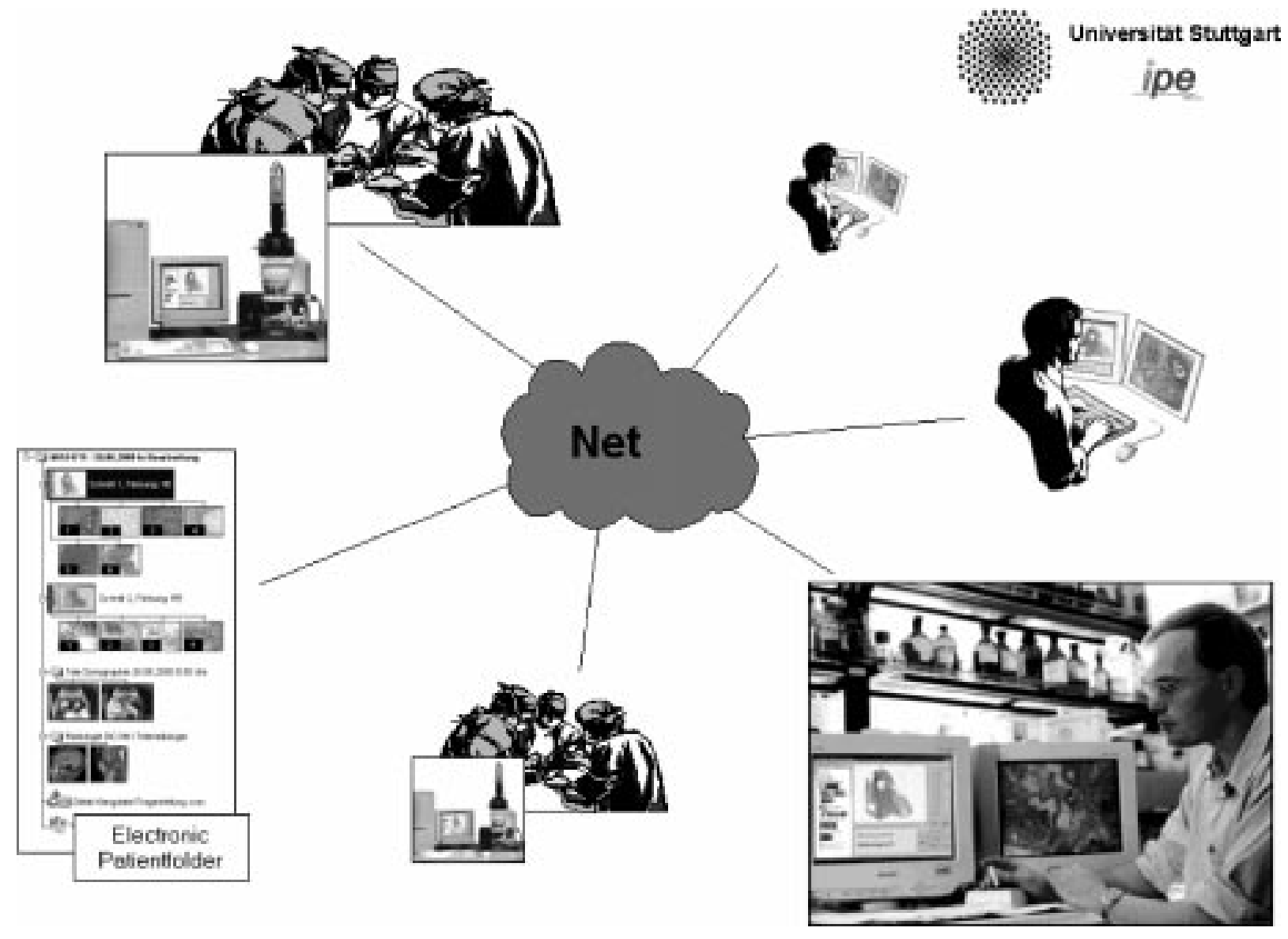

Fig. 8. Future telemedicine networks which will also host telepathology services. 
of service content in their networks, for example, in the DGN (Deutsches Gesundheitsnetz, Bostonnetwork, or the Global Health Care by the Deutsche Telekom AG. The first task for such networks is to overcome the missing interoperability of today's equipment of different manufacturers. It is by far too late to start a formal standardization, to which all manufacturers should comply, because a lot of telepathology equipment is already on the market. A solution to overcome the situation will be to develop interfaces between proprietary software and protocols on the one side and generally accepted standards, which already exist like Websoftware and protocols, on the other side of the software interface. Candidates for such standards may be:

- Image formats (DICOM, Tiff, PDF, GIF, etc.);

- Platform independent software (JAVA, JAVA applets);

- Internet protocols (TCP/IP, Email, FTP, etc.);

- Net interfaces for ISDN, UMTS, Phone, XDSL, Encryption, etc.

The application of components off the shelf reduces the costs for equipment and promotes at least a quasistandardization. Finally there is no doubt that coming telepathology and telemedicine networks will cross national borders at the earliest convenient opportunity

\subsection{Legal aspects of telepathology}

The legal aspects of telepathology have been discussed since 1992 [1,12] and are, in addition, compiled in another contribution of this $A C P$ issue $[1,12]$ and are therefore beyond the present contribution with the exception of the influences of legal aspects on the development of telepathology equipment. For designers and developers of telepathology equipment some considerations of the outcome of the discussions of the legal necessities will be of importance:

Quality, security and safety aspects for equipment: If telepathology equipment becomes operational, in which not each participant is fully informed on all details of the equipment of his partners in a network, he has to rely on a warranty of a minimum standard on the other side of his connection. This is with respect to specimen quality, image quality, transmission and display quality. Means to meet such requirements could be annotation information for the data similar to the DICOM standard in radiology, where images are accompanied by detailed data of the image generation procedure.
New features and developments in telepathology may be initiated by legal recommendations. A candidate could be in the frozen section service telepathology the introduction of haptic sensors and actors - the virtual hand with the virtual telefeeling.

\subsection{What is necessary and what is affordable in telepathology equipment?}

Like in other fields the requirements to telepathology equipment are limited on one side by the state of the art in technology and on the other side by the estimation of the potential users of the affordability of the new technology. In the case of telepathology this will be with respect to the components of the equipment, software, maintenance and fees for telecommunication channels.

Equipment of the store and forward type (expert advice) as well as for teleeducation will rely on already existing microscope, camera and computer equipment; a potential for innovations will be well adapted software.

In telepathology for telediagnosis the community of pathologists more or less unison prefers telemicroscopy to maintain both diagnosis steps: searching for relevant areas in a slide and the diagnosis of found relevant objects.

Components for telemicroscopy add up today to about 50000-70000 $€$. These costs can only be justified if the telepathology application is included into a routine workflow, generating a corresponding cashflow in the pathology department by serving additionally customers, in the surgery department by saving expensive time of the surgery team or in the hospital administration with an increased throughput of patients.

Another consideration for the future acceptance of equipment in daily routine in medicine is the easy (fool proof) operability of a service, because most users are concentrated on the medical problems and not on the machine operation.

\subsection{Market for future components and telecommunication services for telepathology}

Telepathology equipment manufacturers and administrators of telemedicine networks will rely more and more on already existing and especially already accepted and introduced solutions off the shelf. As most of these components have been or are developed not explicitely for the telepathology market, telepathology 
with its only minor economical volume will not influence the technical features of these components. The components will develop according to guidelines of other market segments.

Only components whose market success depends also on telepathology will be influenced by requests from the telepathology community. Examples of this type may be the computer controllable microscopes, scanning stages, telehaptic instruments and teleknifes (if the last two at all are requested by pathologists!). Strong influences however will concern the telepathology software with the user interface and the library of functionality.

Nevertheless some assumptions concerning the future development of key components of telepathology equipment can be made today:

Microscopes. For store and forward telepathology (expert advice) the standard microscope of the pathologist on his desk will be applied also in future; the design of this microscope will reflect the requests of routine pathology. For telemicroscopy presently all international microscope manufacturers offer devices from the upper end of universal microscopes incorporating all modes of microscopy. These instruments include functionality and universality far beyond the necessities of pathology. They are therefore very expensive and cover the main part of equipment costs. From most microscope companies below this level by far less expensive microscopes are offered, which miss just one or the other function necessary for full telemicroscopy. It can be assumed that once the number of telepathology systems ordered reaches a distinct limit, these companies will upgrade these microscopes to the full functionality for telemicroscopy to penetrate the market. Efforts to standardize the computer interface to the microscopes (VMI - Virtual Microscope Interface) [14] will promote the installation of computer controllable equipment.

Scanning stages. Telemicroscopy equipment installs presently scanning stages of the upper end to ensurebe a good positioning and repositioning quality and to avoid artifacts at the border of composite images from tiles. As in the microscope case these universal devices are overdesigned for telepathology. Therefore also in this case by far less expensive scanning stages with reduced universality will appear on the market once its volume reaches a lower limit. An alternative would be to use inexpensive and more inaccurate stages and to compensate the handicaps (coarser steps, limited positioning accuracy) with more intelligent but also more expensive software like substep border fitting of tiles.
Electronic cameras. The development of electronic cameras is currently driven by two markets: TV-related online video cameras with high frame rates and on the other side digital photo cameras with high pixel numbers.

For store and forward applications with no video aspects, the second type is the best selection, because inexpensive digital photo cameras with very high resolutions are already on the market and fit like a normal diapositive camera onto the microscopes. Presently cameras with 4 megapixels are state of the art as, e.g., Megaplus ES:4.0 from Roper Scientific BV, Vianen, The Netherlands; cameras with 16 megapixels enter the market, for example the $4096 \times 4096$ pixels CMOS-chip type KAF-16801 from Foveon Inc. California, USA, or the MegaPlus 16.80i from Roper Scientific BV, Vianen, The Netherlands.

In future telemicroscopy will rely also on the TVrelated camera technology. As pathologists request high quality images, cameras of the 3-chip type are adequate, as each pixel represents all three colors. Presently the 3-chip cameras for normal TV-frames (e.g., $768 \times 576$ pixels TV 3-chip cameras (the DCX 930P from Sony, Tokyo, Japan)) are state of the art, but also cameras with higher resolutions but still maintaining video frequency or near-video frame rates are already on the market (KYF-70 from Victor Company, Tokyo, Japan). They fit and exploit the present resolution of good computer monitors with $1600 \times 1200$ pixels.

Completely new image sensor concepts are investigated and promise also realization in a near future. They rely on semiconductor technology with electronically controllable spectral sensitivity [10]. These sensors will exhibit the advantages of 3-chip cameras (ideal registration of the pixels of the 3 colors) and of the 1-chip cameras (costs, avoidance of the dichroitic mirrors) and will be manufactured in CMOS technology with a superior contrast compared to CCD-type sensors.

Haptic instrumentation. Minimal Invasive Surgery fosters the development of telefeeling to provide the surgeon again with a direct haptic impression of tissue. The technology is in the state of science and still needs some more time to realize products. Devices with the kinematic features of a hand to grip objects are relatively good developed already [13], whereas the sensor part to reproduce not only the forces but also what we call feeling is yet in the beginning. Good ideas to present the signals of the hand and particularly the feeling to the remote partner adequately, meaning to 
provide him with a similar impression, as if he would touch the object himself, are still missing.

It will be the outcome of a longer period of application of today's telemicroscopy equipment to decide if pathologists will request "telesensation" or if the surgeon will overtake this part in the telepathology scenario.

Teleknife. Teleknife technology for the pathologist to make the section remotely (without any telesensation) could be installed today already as the technology is available from telesurgery activities. Since it is a special development for surgery, the costs would be fairly high at the moment, and like in the case of haptic instrumentation only a longer period of application of telemicroscopy can answer the question, if teleknifes should be installed or the activity can be performed by somebody in the surgery team adequately. The question will become more important when new preparation technologies would become state of the art.

Network technology and network services. Store and forward telepathology can already be practiced with today's networks without any restrictions.

The situation is quite different for telemicroscopy, as here the telecommunication channel is the bottleneck for a comfortable working environment. Pathologists ask and will ask in future for higher capacities to transmit online images of highest resolution and detail richness. From other applications will also come stimuli for broader and less expensive telecommunication channels. Only the costs will determine the selection of the adequate telecommunication channels. Service nets in telepathology will be installed as early as pathologists emphasize their interest by purchasing more telepathology systems.

Telepathology software. This paragraph considers only telepathology dedicated software; general PCsoftware like operating system and periphery drivers are standardized products off the shelf.

The software specifically developed for telepathology is and will in the future be the component which is influenced by customers and will decide over success and failure of a telepathology device (see also Section 4.5).

The main challenges for software developers in telepathology can be summarized in following aims:

- Optimization of ergonomy to handle a system.

- Development of intelligent strategies to handle the limited data transmission capacity and to minimize the consequences of it for the operator.
- Considerations of legal requirements like documentation of sessions and supervising of correct system operation.

- Documentation of software for maintenance purposes.

For the three prototype applications of this paper we find three different situations to cover the costs: In teleeducation only a small number of customers is necessary to justify the development costs. Software costs in this case can be kept low if on the clients site only standard browser software is requested. Then only for the server site special software has to be developed. Clients can contribute to the server service by sending contributions (e.g., by email) to the server institution, where it will be integrated in the already existent server environment for access to all other participants.

In interactive telemicroscopy the software will be the most important part. In this application the aspects of ergonomy, handling of the telecommunication channel and in the near future special software to evaluate images are the most prominent. The software will include blocks for the remote microscope operation, slide handling, data transmission, data and image presentation and finally add-on services like image processing and interpretation.

Interoperability between telemicroscopy systems of different manufacturers will become a challenging effort for general telepathology networks; today only for a few blocks interoperability steps are under development [14].

\subsection{Mutual influences of system properties and application experiences on future developments}

Besides the technical improvement of components and of telepathology system software, mainly the mutual influences between existing operational systems and experiences gained using them in daily routine workflow, together with ideas to install new service types will drive the development and the catalog of facilities of next generation devices. Figure 9 may illustrate this cycle.

Present generation equipment especially of the telemicroscopy type and the experiences gained in longer operational periods revealed a collection of requests and suggestions for the next generation devices for the three prototypes: 


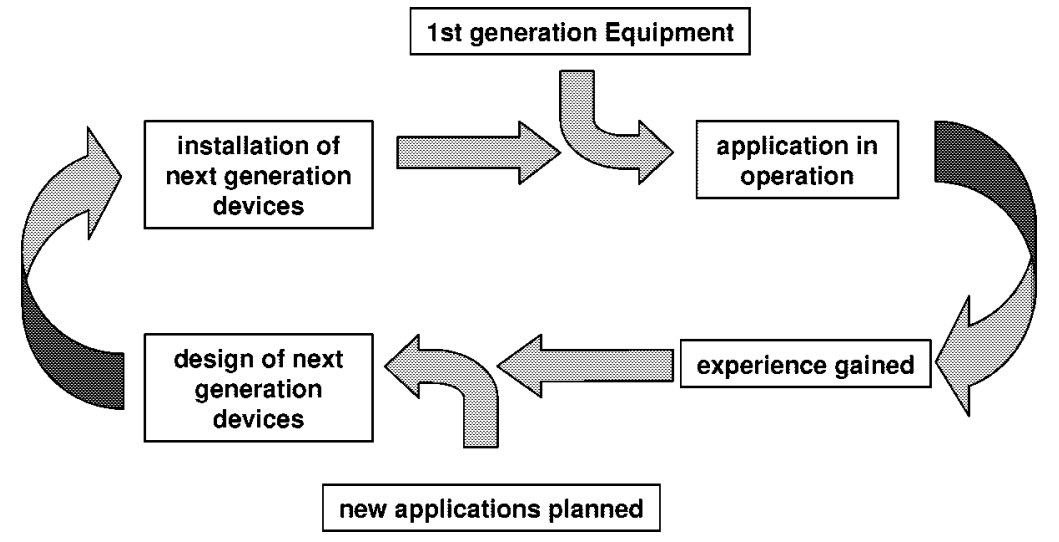

Fig. 9. Mutual influence of existing equipment and experiences gained with it on the development of next generation devices.

\subsubsection{Teleeducation}

Most relevant for the continuing development of this type of telepathology will be:

- How many pathologists are interested in continuing education and how many students will accept telepathology as a training tool? May telepathology become a tool for quality control programs in pathology? If the interest in participation is sufficiently large, the next step will be to install relevant data bases with annotated image material and case documentation well suited for education. The format of this information has to meet a general accepted standard.

- The technology of such education networks should be of the client server type. For the clients standardized every where already installed software should be applied as for instance Web browsers and Java applets transmitted from the server.

- On the server location sufficient efforts should be undertaken to develop new didactic concepts well adapted to telelearning. Sufficient resources must be planned to operate and maintain such teleeducation centers.

- The hard- and firmware components will come still from the PC-market.

- Pioneers in the field already have begun to install such data bases for several fields in pathology, e.g., for cytology and for hematology.

\subsubsection{Store and forward telepathology for expert advice applications}

In this category the impulses for the next generation of hard- and software will be influenced from the outcome of the experiences of operational equipment:

- As before, the main influence will be the acceptance and requests for expert advice telepathology on a sufficiently large scale. If this is the case professional and certified software for a broad routine application will appear on the market.

- The hard- and firmware will consist of components from the general computer and microscope market.

- Experiences for the development of the next generation devices will come from more or less already official operational services as, e.g.,

* The AFIP network of the American Army.

* The experiences of the EUROQUANT server in Dresden for DNA histogram measurement and interpretation of cytologic cell specimens.

* The starting network of the UICC for expert advice telepathology in oncology operated by the Charité in Berlin [2].

* Software has to be designed to glue together single images to case folders in a standardized structure adequate for the tissue involved. Also this software should be compatible with concepts of other telemedicine applications like radiology and hospital information systems.

* Screening of cytological slides can be performed with store and forward equipment. For diagnosis in this case all fields of view containing cells have to be transferred. Normally (e.g., in PAP-screening) this will lead to tremendous amounts of data and to the corresponding unacceptable transfer times. A way out for PAPscreening is the employment of a cytotechnician or the installation of a prescreening machine, which selects automatically diagnostic important cells and transfers only those to the cytopathologist. 


\subsubsection{Telemicroscopy}

Telemicroscopy requires fairly complex equipment and software and must be designed also for the most challenging application in pathology, the intraoperational frozen section service. Therefore the developers of next generation devices will be confronted with many suggestions and requests from the users community:

- As before the acceptance and introduction of telemicroscopy services in telepathology will provide the main argument for investments in new software versions. Especially the frozen section service comprises a high economical potential and will drive the field. Therefore the main influences will come from recommendations of national professional chambers and legal issues imposed on the properties of future equipment for telediagnosis. Another problem of this type is the redistribution and definition of tasks in pathology, if frozen section preparations are produced in the surgery department without the physical presence of a pathologist as already discussed in the beginning of the paper.

- Related to the point above is the question, to which extent frozen section diagnosis services will change, when they become available more easily at the surgeons operation theatre and when new intraoperational diagnostic opportunities will develop.

- New aspects in telepathology in future may be services providing image processing and evaluation facilities remotely (as, e.g., the DNA histogram service of the Dresden group).

- What will be the effect of increasing specialization in pathology? Will 2nd diagnosis become a relevant service?

- From a technical point of view, as well as from the users, the bottleneck of data transmission rates of affordable telecommunication services will stay a continuing challenge for developers.

- The item above is a key for the application of telemicroscopy in screening cytology applications. Due to huge numbers of fields of view (full inspection of a slide with high magnification) the time needed for routine screening, e.g., of PAPsmears is prohibitive. The way out is only the application of broadband telecommunication channels or a preselection of objects of interest at the consulting partners location as in the case of expert advice devices (e.g., prescreening machines). Obviously this argument is not valid for slides with only few cells.
- First tests for a new application of telemicroscopy in quality control of services in pathology have been carried out. In this test pathologists in the UK, who have to undergo a biannual test in diagnosing test preparations in histology, were asked to make their test in a telemicroscopy environment to avoid in future sending around slides with a high risk of loss and damage plus an extremely long time to get the results. If such an application is accepted, this would be the entrance of telemicroscopy in realistic teleeducation for diagnosing slides with both activities: searching for relevant events and diagnosing the found objects.

- How big will be the pressure to form larger competent telepathology networks? This would increase the efforts to develop tools to make equipment and services interoperable between institutions and equipment manufacturers. This depends on tendencies in pathology to form small islands of networks between cooperating colleagues on one side and to join large service networks on the other side.

- The introduction of telemicroscopy in a daily workflow in pathology will request immediately tools to interface pathology to the hospital information system (HIS) and to other departments in a hospital involved in treatment of the same patient group.

\section{Conclusions}

Today's telepathology equipment has reached a state in technology enabling an integration in routine workflow. This holds perfectly for teleeducation and expert advice equipment, where all requests of pathologists can be met. In teleeducation the only problems left are adequate contents and didactic forms for large and efficient education programs. In expert advice telepathology, many pathologists accept even equipment below the state of the art.

In online telediagnosis two problems for ideal devices still remain: the haptic telepresence of the pathologist and an increased cheap data transmission channel to further improve the online impression.

Especially in the frozen section scenario some legal questions, which arise due to the change in workflow, have to be settled between surgeons and pathologists.

For all types of telepathology, operational equipment is commercially available. The near future decides upon the acceptance of the new tools. 
After a first operational period with existing equipment, experiences gained in daily workflow will initiate the next software generation, which will run on the same equipment.

The first generation of telepathology users also will decide, if telepathology will be applied in the future between partners or within general telemedicine networks offering an open market for services offered and requested in pathology.

The degree of integration of further services into the platforms of telepathology equipment, the forming of networks and the integration of pathology workstations and telepathology devices may become an interesting development in the near future.

\section{References}

[1] C. Dierks, Legal aspects of telepathology; in the same $A C P$ issue.

[2] M. Dietel, P. Hufnagl and T.N. Nguyen-Dobinsky, The UICC telepathology consultation center - a global approach to improving consultation for pathologists in cancer diagnosis, Cancer 89 (2000), 187-191.

[3] K. Kayser, G. Kayser and S. Zink, New technical aspects in telepathology, Elec. J. Pathol. Histol. 6(3) (2000), No. 003-04.

[4] K. Kayser and G. Kayser, Basic aspects of and recent development of telepathology in Europe with specific emphasis on quality assurance, J. Anal. Quant. Cytol. Histol. 21 (1999), 319-328.
[5] K. Kayser, Telemedizin, Wiener Klin. Wschr. 108 (1996), 932-940.

[6] K. Kayser, J. Szymas and R. Weinstein, Telepathology, Electronic Communication, Education and Publication in Pathology, Springer-Verlag, Berlin, Heidelberg, 1999.

[7] R. Lenz, Digitale Kamera mit CCD-Flächensensor und programmierbarer Auflösung bis zu $2994 \times 2320$ Bildpunkten pro Farbkanal; Informatik Fachber, in: Proc. 11. DAGMSymposium 1989, Springer-Verlag, Hamburg, 1989.

[8] J. Leong, A. Graham, T. Gahm and J. McGee, Telepathology: Clinical utility and methodology, Recent Advances in Histopathology 18 (1999).

[9] MountainView ${ }^{\mathrm{TM}}, \mathrm{CMOS}$ camera $1024 \times 1024$ pixel, MessTec \& Automation (1 Sept. 2000), 26.

[10] T. Neidlinger, C. Harendt, J. Glöckner and M.B. Schubert, Novel device concept for voltage-bias controlled color detection in amorphous silicon based CMOS cameras, Mat. Res. Soc. Symp. Proc. 557 (1999).

[11] H.S. Sawhney, S. Hsu and R. Kumar, Robust video mosaicing through topology inference and local to global alignment, in: Computer Vision - ECCV'98, Freiburg, June 1998, Lecture Notes in Computer Science, Vol. 1407, Springer, pp. 103-119.

[12] M. Schiffer, Legal aspect of telepathology, Zetralbl. Pathol. 138 (1992), 393-394.

[13] S. Schulz, Ch. Pylatiuk and G. Bretthauer, A new class of flexible fluidic actuators and their applications in medical engineering, Automatisierngstechnik 47(8) (1999), 390-395.

[14] P. Schwarzmann, Interoperability report (version 3.3), May 1999, European Union, project EUROPATH (DG XIII-Project HC 1038). 


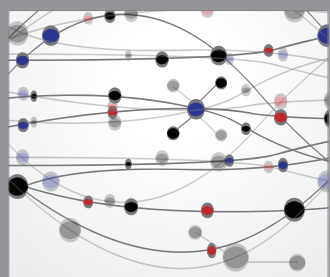

The Scientific World Journal
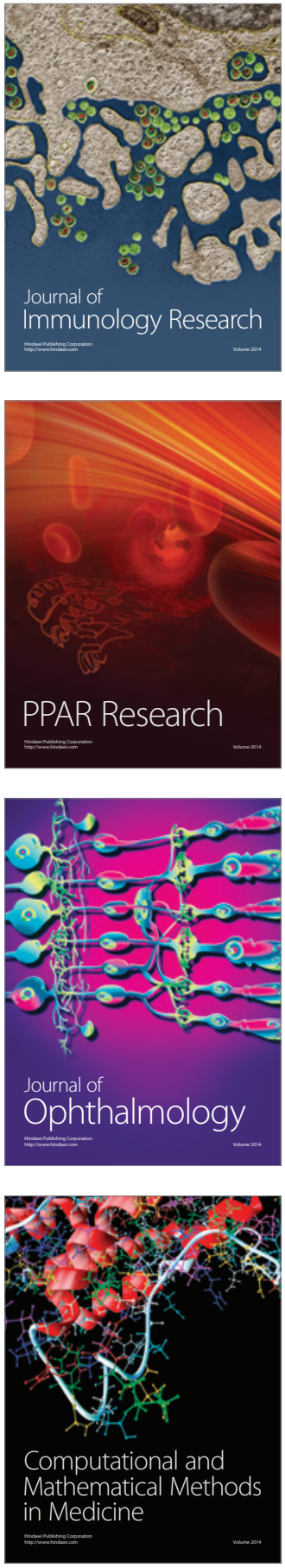

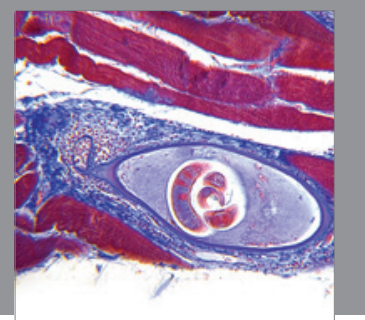

Gastroenterology

Research and Practice
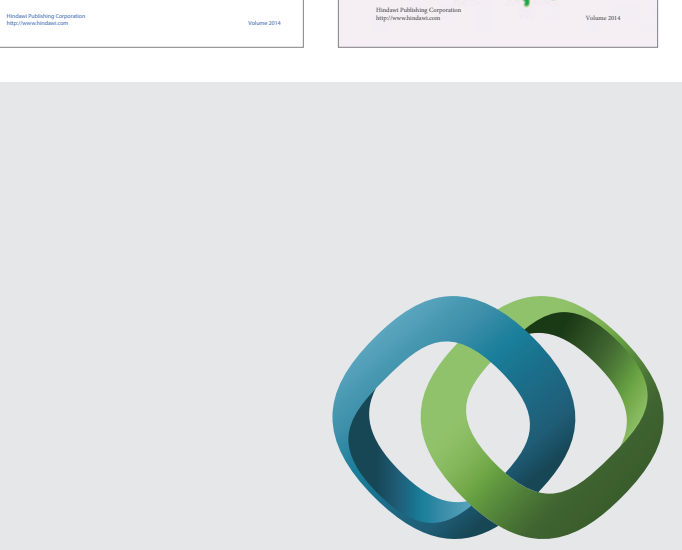

\section{Hindawi}

Submit your manuscripts at

http://www.hindawi.com
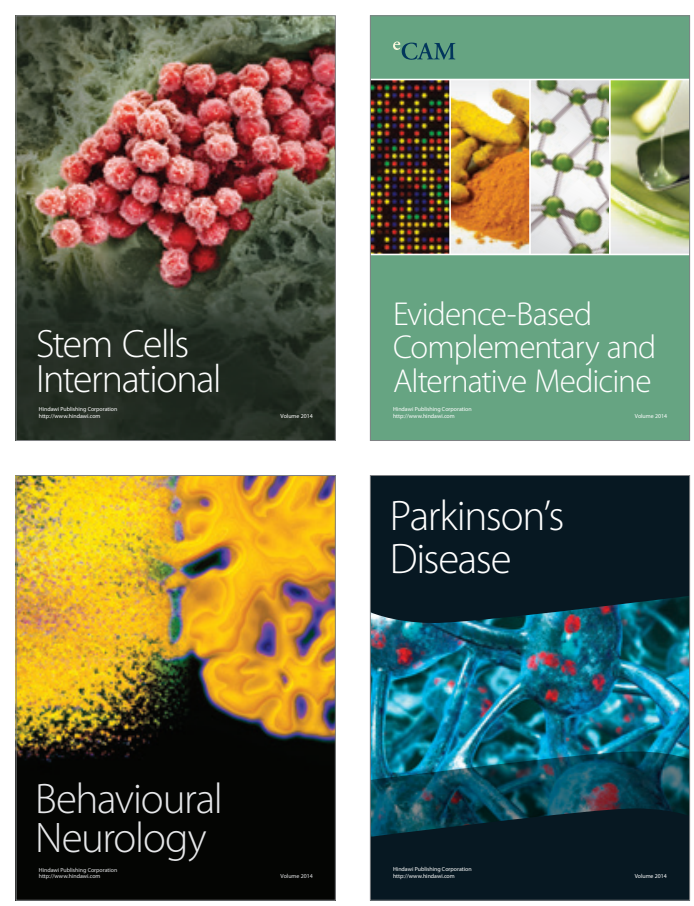

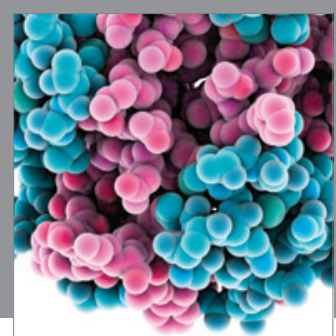

Journal of
Diabetes Research

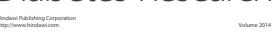

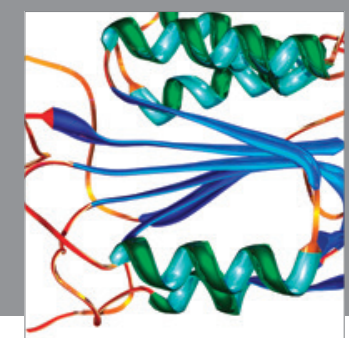

Disease Markers
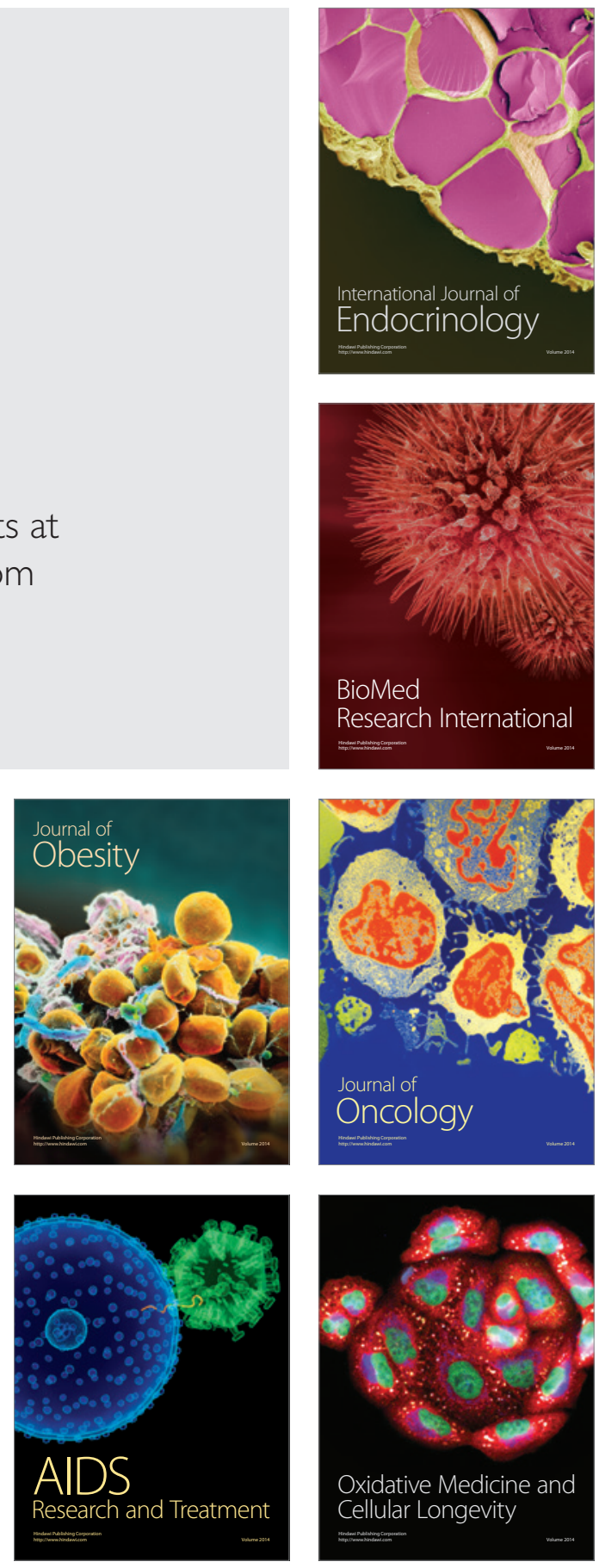